\title{
Utilização de Modelos Mentais na Representação de Estruturas Cognitivas de Aplicativos TVDi
}

\author{
Alex de S. Vieira1, Rosendy J. F. Galabo², André L. Demaison ${ }^{3}$, Carlos de S. Soares \\ Neto 4 \\ ${ }^{1}$ Faculdade de Computação e Engenharia Elétrica (FACEEL) \\ Universidade Federal do Sul e Sudeste do Pará (UNIFESSPA) - Marabá - PA - Brasil \\ ${ }^{2}$ ImaginationLancaster \\ Lancaster University - Lancaster, U.K. \\ ${ }^{3}$ Departamento de Informática (DEINF) \\ ${ }^{4}$ Departamento de Desenho e Tecnologia (DEDET) \\ Universidade Federal do Maranhão (UFMA) - São Luís, MA - Brasil \\ alexvieira@unifesspa.edu.br, r.j.galabo@lancaster.ac.uk, \\ andreleonardo@gmail.com, csalles@deinf.ufma.br
}

\begin{abstract}
In the context of interactive digital TV applications (iDTV), mental models can be especially useful to represent the cognitive structure of users during learning these applications. These models help to highlight which elements (e.g. behavioral and spatial) are perceived by users, thus contributing to a proper understanding and use of learning objects, created for iDTV environment through metacommunication between developers and users. The results of this study show that the use of metaphors as storyboard and natural language are recurrent among users and that the elements of an interactive narrative for iDTV are not yet fully understood by beginners users.
\end{abstract}

Resumo. No contexto de aplicativos TV Digital interativa (TVDi), modelos mentais podem ser especialmente úteis para representar a estrutura cognitiva de usuários durante o aprendizado desses aplicativos. Tais modelos ajudam a destacar que elementos (e.g. comportamentais e espaciais) são percebidos pelos usuários contribuindo, assim, para uma adequada compreensão e uso de Objetos de Aprendizagem, criados para o ambiente TVDi, por meio da metacomunicação entre desenvolvedores e usuários. Os resultados deste estudo revelam que o uso de metáforas como storyboard e linguagem natural são recorrentes entre os usuários e que os elementos de uma narrativa interativa para TVDi ainda não são plenamente compreendidos por usuários iniciantes.

\section{Introdução}

Os modelos mentais fundamentam-se no fato de o ser humano captar o mundo indiretamente, isto é, por meio de modelos. Esse conceito pode ser aplicado em diversas áreas do conhecimento - o que tem favorecido sua adoção pela Psicologia Cognitiva Contemporânea, Interface Homem-Computador, educadores e pesquisadores de outras áreas do conhecimento. Tal conceito baseia-se na teoria da aprendizagem significativa apresentada em (TAVARES, 2006) e (MOREIRA, 2000), que abrange uma série de princípios que valorizam a construção do conhecimento por meio de representações idiossincráticas utilizadas para representar sistemas reais presentes em nosso cotidiano. 
V Congresso Brasileiro de Informática na Educação (CBIE 2016)

Anais do XXVII Simpósio Brasileiro de Informática na Educação (SBIE 2016)

De maneira direta, um modelo mental é um modelo criado na mente de um participante que o permita manipular um conjunto de diferentes elementos (imagens, palavras, etc.) e propriedades (causalidades, relacionamentos, etc.) que são utilizadas para representar a realidade por meio de analogias a sistemas reais (BORGES, 2008) como, por exemplo, o funcionamento de uma cafeteira ou ainda sistemas abstratos como o funcionamento de programas TVD $i$ - este último é objeto de estudo desta pesquisa e é discutido a seguir.

Aplicativos TVD $i$ são programas que possibilitam ao usuário escolher um fluxo audiovisual alternativo, dentre vários disponíveis, em um programa de TV interativa. Esse tipo de programa é caracterizado pela sincronização de mídias audiovisuais com outras mídias, cuja sequência de execução é controlada pelo usuário que a manipula, usualmente, por meio de um controle remoto. E Objetos de Aprendizagem (OA) são quaisquer entidades de mídias digitais (games, aplicativos, web sítios, etc.) ou materiais que podem ser (re)utilizados ou referenciados no processo de ensino (DAMASCENO et. al., 2014) contemplando mídias como, por exemplo, textos, vídeos, áudios e fotos.

Para que não haja dúvidas quanto a polissemia da palavra programa usada neste trabalho, faz-se necessário definir um programa de TV como um conteúdo audiovisual linear (que possui começo, meio e fim bem definidos) veiculado por uma emissora, isto é, trata-se de uma atração televisiva que não permite o usuário interagir com o conteúdo.

Um aplicativo TVDi (ou aplicação TVD $i$ ) é um tipo de programa como um programa de computador que é adicionado ao programa de TV, dotando-o de possibilidade de interação. Um programa TVD $i$, por sua vez, pode ser entendido como a junção de um aplicativo TVD $i$ e um programa de TV, ou seja, é um programa de TV cujo conteúdo audiovisual linear é transformado em um conteúdo não-linear. Observa-se que, neste experimento, o termo Objeto de Aprendizagem (OA) - ou OABVI, explicado adiante - corresponde a um programa TVD $i$, cujo vídeo principal equivale a narrativa interativa.

Neste trabalho, foram empregados métodos da pesquisa qualitativa e quantitativa com o intuito de verificar a representação de aplicativos TVD $i$ por meio de modelos mentais elaborados por alunos do curso superior de Design, de uma universidade pública federal brasileira. $\mathrm{O}$ foco desta pesquisa é a verificação de estruturas cognitivas inerentes a aplicativos TVD $i$, a partir dos modelos mentais criados por discentes do ensino superior.

Nesse processo é possível observar a metacomunicação (BARBOSA, 2010) efetiva entre equipe de desenvolvimento e usuário. Isto é, enquanto de um lado da comunicação há uma interface composta por diferentes objetos de mídias elaborada por uma equipe desenvolvedora, por outro lado, tem-se o usuário final (representado por discentes participantes do teste) comunicando-se por meio de signos e mensagens da interface com a referida equipe.

A partir deste trabalho espera-se que desenvolvedores de aplicativos TVD $i$ se apropriem dos resultados para elaborarem aplicativos cada vez mais voltados aos principais elementos e funções percebidas pelos usuários, mediante seus respectivos modelos mentais, de modo que interfaces mais significativas sejam desenvolvidas, quer sejam para aplicativos ou ferramentas de autoria para TVD $i$.

Este trabalho está organizado da seguinte forma. Na Seção 2 encontram-se os trabalhos relacionados. A Seção 3 apresenta a metodologia empregada, ressaltando o 
V Congresso Brasileiro de Informática na Educação (CBIE 2016)

Anais do XXVII Simpósio Brasileiro de Informática na Educação (SBIE 2016)

desenvolvimento de um OA voltado a TVDi, o ambiente de teste e os principais pontos investigados. Na Seção 4 encontram-se os resultados e suas respectivas análises. Por fim, na Seção 5 encontram-se as considerações finais e trabalhos futuros.

\section{Trabalhos Relacionados}

Em Edwin et. al. (2012) é proposto um sistema baseado em aprendizagem multiplataforma voltado ao ambiente de TVDi e contempla o processo de criação de Objetos de Aprendizagem Baseados em Vídeos Interativos (OABVI). O trabalho faz uso da corrente pedagógica construtivista utilizando de fóruns de discussão e do enriquecimento de vídeos inicialmente despidos de interatividade.

Neste trabalho foi desenvolvido um aplicativo que, assim como em Edwin et. al. (2012), também consiste de um OABVI, todavia seu foco é meramente ilustrativo e tem por finalidade nortear os discentes participantes do teste quanto a elementos comportamentais, estruturais e espaciais de um aplicativo TVDi. Em outras palavras, foi desenvolvido um OA utilizando um vídeo, inicialmente, linear que foi enriquecido de outras mídias para torna-lo interativo.

Chaves et al. (2011) desenvolveu um game chamado de DesiGMPS, voltado ao ensino de Modelos de Qualidade de Processo de Software. Nesse game o Mapa Conceitual (MC) foi utilizado como recurso de verificação de aprendizagem de conceitos e relacionamentos referentes ao Processo de Melhoria de Software (MPS). O objetivo principal desse trabalho era verificar se, após o uso do game, o discente conseguiria dominar conceitos e estabelecer adequadamente os seus inter-relacionamentos.

Similarmente a proposta deste trabalho, Chaves et al. (2011) organizou os elementos de sua proposta em dois grupos principais (conceitos e relacionamentos) conferindo-lhes identificadores, descrição e pesos diferentes e, depois de calcular uma medida que chamou de "medidas de similaridades locais" (MSL), foi calculada a similaridade geral (SG) que confere a pontuação do aluno e verifica a possibilidade de mudança de fase. Segundo os resultados apresentados, 74\% dos participantes informaram que a disciplina tornou-se mais interessante e que esse OA deveria ser utilizado em outras disciplinas $(95 \%)$.

De acordo com Damasceno et. al. (2014) o DesiGMPS pode ser considerado um OA. Contudo, diferente de Chaves et al. (2011) neste trabalho foram estudados os modelos mentais dos participantes para verificar que estruturas seriam particularmente reconhecidas por eles frente ao uso de um OA voltado a TVD $i$. A diferença principal é que esses modelos têm caráter mais livre do que os $\mathrm{MC}$ - os quais se constituem em uma formalização genérica dos modelos mentais e, portanto, foi necessária a criação de uma proposta específica para a avaliação dos pesos de cada elemento percebido pelo discente.

\section{Metodologia}

Nesta seção são apresentados o protótipo de alta fidelidade e alguns princípios e questionamentos utilizados como métricas para a obtenção e avaliação dos resultados.

\subsection{Roteiro do Teste}

Incialmente foi desenvolvido um protótipo de alta fidelidade (MOREIRA e SANTA ROSA, 2012) para um programa TVD $i$, o qual foi utilizado para demonstrar os principais 
V Congresso Brasileiro de Informática na Educação (CBIE 2016)

Anais do XXVII Simpósio Brasileiro de Informática na Educação (SBIE 2016)

conceitos (links, mídias, regiões, descritores, conectores, etc.) relacionados a aplicações interativas no contexto de TV Digital. A implementação desse protótipo justificou-se pela necessidade de apresentar o aplicativo e depois de avaliar a compreensão desse aplicativo pelos usuários finais.

No segundo momento, 18 alunos do curso de graduação regularmente matriculados no $2^{\circ}$ período do curso de Design, foram convidados a assistirem uma aula correspondente à demonstração do protótipo para que, em seguida, tentassem (individualmente) explicar o programa TVD $i$ expressando livremente os conceitos assimilados e que utilizassem, para isso, apenas papel e caneta. Esses participantes foram selecionados por deterem um conhecimento ainda pouco consolidado sobre os conceitos verificados tratando-se, portanto, de um perfil intermediário entre um usuário comum (sem conhecimentos específico no assunto) e um acadêmico especializado.

Os conceitos apresentados no primeiro momento foram identificados nos modelos mentais por meio de um framework de critérios específicos para este trabalho. Esses critérios possuem rótulos e pesos diferentes - sendo explicado mais adiante nesta seção. Além disso, uma abordagem qualitativa também foi considerada na avaliação dos modelos.

\subsection{Descrição do Protótipo}

O protótipo consiste de um aplicativo TVDi que exibe um ícone de cor vermelha no canto direito superior da tela (indicando a presença de conteúdo interativo) no mesmo instante em que o vídeo principal é iniciado, conforme apresentado na Figura 1.

O storyboard interativo da Figura 1 esquematiza os possíveis relacionamentos entre as cenas. Assim, quando o botão vermelho do controle remoto é selecionado, uma informação na base da tela deve ser exibida, conforme mostra a Figura 1(b) e o ícone vermelho anteriormente apresentado deve desaparecer. Nesse momento, caso o botão vermelho do controle seja novamente selecionado, então o conteúdo adicional presente na Figura 1(b) deve dar lugar a Figura 1(a). Por fim, ao terminar o vídeo principal, quer esteja na situação (a) quer na situação (b), o aplicativo deve ser encerrado (o que é simbolizado pela imagem da ação stop na extremidade direita da figura).

O sincronismo entre as mídias (vídeo, imagem e texto) do aplicativo é orquestrado por meio de relacionamentos causais, os quais são descritos na linguagem NCL (Nested Context Language) (SOARES e BARBOSA, 2012) e expressam o comportamento final apresentado aos alunos na forma de programa TVD $i$.

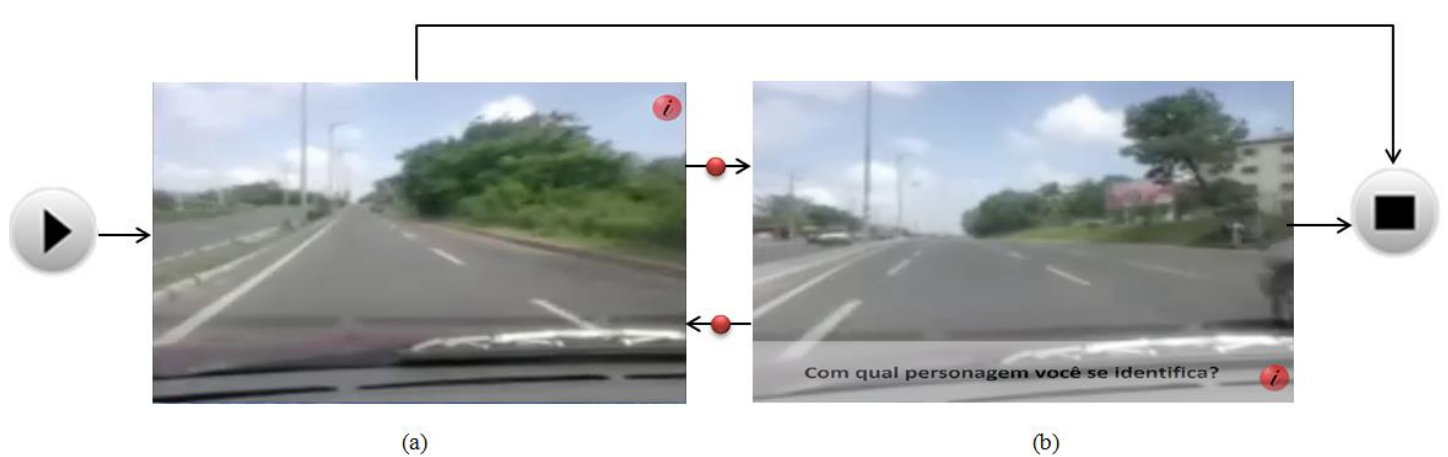

Figura 1. Storyboard interativo do programa TVDi 
V Congresso Brasileiro de Informática na Educação (CBIE 2016)

Anais do XXVII Simpósio Brasileiro de Informática na Educação (SBIE 2016)

A Figura 2 apresenta um trecho de código NCL extraído do protótipo. Ela exemplifica a forma como um desenvolvedor (especialista) expressa esses relacionamentos entre as mídias.

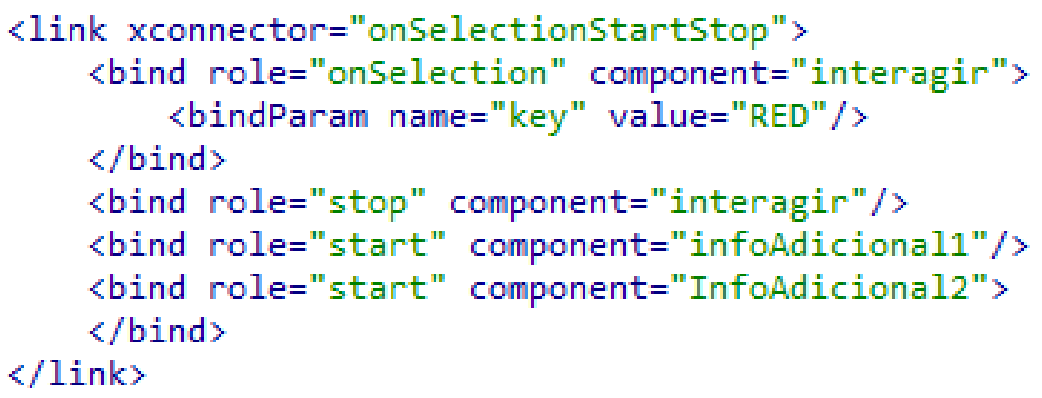

Figura 2. Relacionamento expresso em NCL

O relacionamento (ou link) observado na Figura 2 representa a possibilidade de selecionar o botão vermelho (tag: "bindParam name = "key" value $=$ "RED") enquanto o ícone chamado de "interagir" estiver em exibição e, caso essa seleção ocorra, o conteúdo adicional ("InfoAdicional1" e "InfoAdicional2") deve ser iniciado e o ícone "interagir" deixa de ser apresentado na tela. O entendimento do comportamento desse link é importante, pois a sua lógica se estende aos demais, cuja conjuntura expressa, em linguagem declarativa, o modelo mental do especialista e trata-se de um referencial para a obtenção dos demais modelos mentais.

\subsection{Framework de Critérios Avaliativos}

Com o objetivo de coletar e mensurar os dados algumas questões apresentadas em (TAVARES, 2006) foram adaptadas a alguns conceitos da linguagem NCL. Essa adaptação é especialmente útil para auxiliar no processo de sistematização dos resultados.

As perguntas a seguir compõem o framework de critérios utilizados para a identificação e avaliação dos principais conceitos associados aos modelos mentais da prototipagem. Como funciona o programa TVD $i$ ? (verificar se o participante consegue descrever o sistema); O que o sistema está fazendo? (Prever ou explicar o estado do sistema); O quê o programa manipula? (reconhecer a natureza multimídia do aplicativo); Onde são exibidas as mídias? (identificar a noção de distribuição espacial das mídias); Quando as mídias são executadas? (verificar o momento em que acontece o sincronismo entre duas ou mais mídias); Como elas (mídias) são exibidas? (verificar como as mídias são exibidas na tela).

Neste trabalho, cada critério observado está rotulado em um dos três grupos: NI Não informado (quando o participante não faz menção ao conceito avaliado); PI Parcialmente informado (quando o participante faz uma referência imprecisa a um conceito - tendo como espelho a linguagem utilizada pelo especialista - ou quando um conceito é utilizado sem explicação adicional; e EI - Explicitamente informado (quando há no modelo mental uma indicação clara de determinada parte do sistema, ainda que em menor grau de ocorrência). A Tabela 1, a seguir, sintetiza essas classificações, contudo deve-se assumir os valores 1, 2 e 3 respectivamente para os grupos NI, PI e EI.

Para diferenciar cada os participantes $(\mathrm{P})$ foi adotado uma numeração associada a cada um deles. Assim, P1 refere-se ao participante 1; P2 refere-se ao participante 2; P3 
V Congresso Brasileiro de Informática na Educação (CBIE 2016)

Anais do XXVII Simpósio Brasileiro de Informática na Educação (SBIE 2016)

refere-se ao participante 3 e assim sucessivamente. A Tabela 1 apresenta a divisão dos critérios utilizados para classificar os modelos mentais gerados de acordo com o grupo e com o peso: 1. utilização do conceito e 2. descrição do conceito.

\begin{tabular}{|c|c|c|}
\hline 1. Utilização do Conceito & Rótulo & Peso \\
\hline Mídia & NI / PI / EI & $1 / 2 / 3$ \\
\hline 2. Descrição do Conceito & Rótulo & Peso \\
\hline Descrição do programa TVD $i$ & NI / PI / EI & $1 / 3 / 5$ \\
\hline
\end{tabular}

Tabela 1. Framework de critérios avaliativos

A primeira classificação (utilização do conceito) refere-se a conceitos isolados como, por exemplo, mídia e relacionamento, cujo objetivo principal é identificar se o participante os emprega em seu modelo mental. A segunda classificação (descrição do conceito) permite investigar se o participante compreende o programa que está sendo investigado e se ele consegue explicá-lo ou descrevê-lo.

Como pode ser observado na Tabela 1 , há uma diferença na distribuição dos pesos. Isso se explica devido ao fato dos modelos mentais terem como premissa a necessidade de explicar sistemas por meio de modelos particulares e não apenas de identificar os elementos que compõem esses sistemas. Por isso os pesos dos rótulos relacionados à utilização dos conceitos contêm valores menores que os pesos rotulados para a descrição do conceito, ou seja, a descrição denota um maior domínio de compreensão do que a alocação de um conceito ou propriedade.

Vale informar também que os modelos mentais podem ser muito arbitrários e variam conforme a carga cognitiva de cada participante e que - até o desenvolvimento deste trabalho - não foi identificado um cálculo formal para quantificar esses modelos, ainda assim, neste trabalho, foi desenvolvido um cálculo próprio visando a geração de estatísticas para que os resultados pudessem ser melhor interpretados pelos autores.

Dito isso, para calcular a pontuação média de modelos mentais relacionados a aplicativos TVDi, deve-se considerar uma atribuição prévia de valores aos rótulos NI = $1, \mathrm{PI}=2$ e $\mathrm{EI}=3$ (tanto para utilização do conceito quanto em descrição do conceito). Os pesos 1, 2 e 3 (valores referentes a utilização de conceitos, propriedades ou relacionamentos) e 1, 3 e 5 (valores referentes a descrição dos conceitos, propriedades ou relacionamentos).

$$
\mathrm{M}=\sum_{\mathrm{i}=1}^{9} \mathrm{U}=\frac{(\mathrm{VRU} \times \mathrm{PRU})+\sum_{\mathrm{i}=1}^{10} \mathrm{D}=(\mathrm{VRD} \times \mathrm{PRD})}{\mathbf{2}}
$$

Equação 1. Fórmula para calcular a pontuação média do modelo mental Em que:

$\mathrm{M}=$ Pontuação Média do modelo mental;

$\mathrm{U}=$ Média referente a Utilização do conceito

- VRU = Valor médio do Rótulo de Utilização;

- PRU = Peso médio do Rótulo de Utilização; 
V Congresso Brasileiro de Informática na Educação (CBIE 2016)

Anais do XXVII Simpósio Brasileiro de Informática na Educação (SBIE 2016)

$\mathrm{D}=$ Média referente a Descrição do conceito

- $\mathrm{VRD}=$ Valor rotulado de descrição; e

- PRD = Peso rotulado de descrição.

Observa-se que os conceitos classificados como "não informados" (grupo NI) recebem um peso mínimo, que não compromete o resultado final do cálculo, dado que seu valor equivale a 01 (um) e, assim, quando posto na fórmula em nenhuma hipótese resultará em valor negativo ou nulo.

Assim, a pontuação média do modelo mental $(\mathrm{M})$ do participante é o resultado da média de utilização dos conceitos (U) (composta pelos VRU multiplicado pelo PRU) somado com a média das descrições dos recursos (D) (composta pelos VRD multiplicado pelo PRD) cujo valor final fora dividido por 02 (dois), dado que foram consideradas duas bases avaliativas (utilização e descrição conceitual). De maneira geral, a média do modelo mental do usuário é dada por $\mathrm{M}=(\mathrm{U}+\mathrm{D}) / 2$.

É importante considerar que, nesta avaliação, a linguagem natural também é considerada válida e pode ser enquadrada em um dos rótulos informados anteriormente, sem qualquer decréscimo na sua caracterização ou peso, posto que a diferença reside simplesmente na forma (verbal ou pictórica) de como o conceito é expressado no modelo mental, isto é, podem ser realizada uma descrição textual no lugar elementos visuais para expressar, por exemplo, a existência de links (relacionamentos) e objetos de mídia - essa estratégia foi utilizada por alguns participantes.

\section{Resultados e Discussões}

Dos 18 modelos mentais selecionados, 05 não foram avaliados neste trabalho porque refletem a representação de um software de criação de um programa TVD $i$, distanciandose da proposta original que é a representação de um programa de TV interativo propriamente dito.

Nesta seção são apresentados os resultados gerados da análise de 13 participantes.

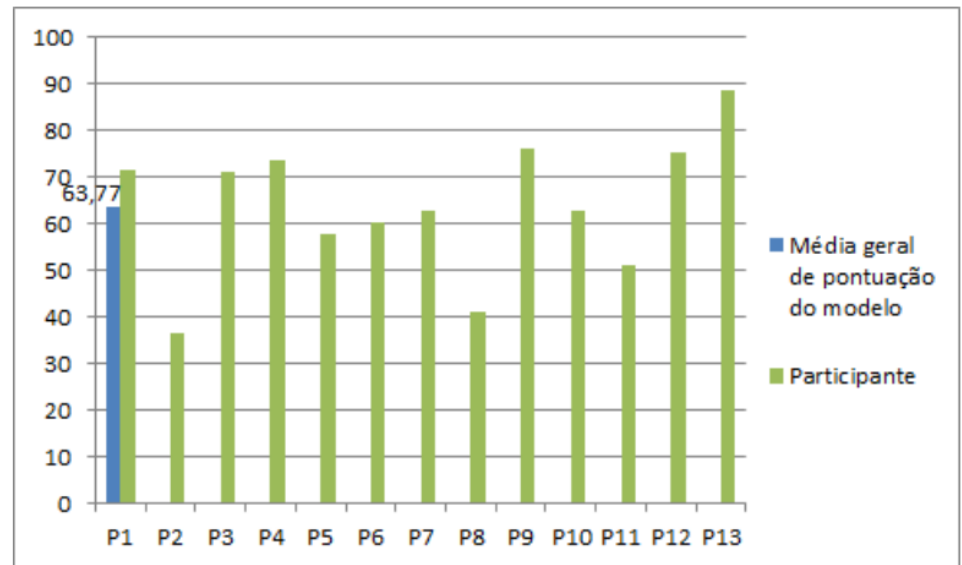

Figura 3. Comparativo da pontuação média dos modelos mentais

A partir do cálculo da pontuação média discutido na seção anterior, foi encontrado o valor de 63,77 que representa a média geral dos pesos atribuídos aos modelos mentais dos participantes. A Figura 1 é um gráfico que estabelece um comparativo entre as médias individuais (eixo horizontal) dos participantes e a média geral (eixo vertical). 
V Congresso Brasileiro de Informática na Educação (CBIE 2016)

Anais do XXVII Simpósio Brasileiro de Informática na Educação (SBIE 2016)

Quanto ao emprego dos conceitos, a taxa média foi de 53,8 e notou-se que 08 participantes apresentaram resultados acima da média, enquanto os 06 restantes tiveram uma pontuação abaixo da média. Com relação à descrição dos conceitos, a pontuação obtida foi de 73,8 . Nessa categoria, 07 participantes tiveram uma pontuação abaixo da média, porém os demais conseguiram uma pontuação que ultrapassa em mais de 13 pontos a média.

Quanto a conjuntura dos modelos gerados, pôde-se observar que todos os participantes utilizaram-se da linguagem pictórica para estruturar o comportamento do aplicativo TVDI, como pode ser observado em um exemplo apresentado na Figura 4. A estrutura dinâmica do aplicativo corresponde à estrutura cognitiva que o participante cria para simular o aplicativo interativo e pode servir de base para representar o seu modelo mental.

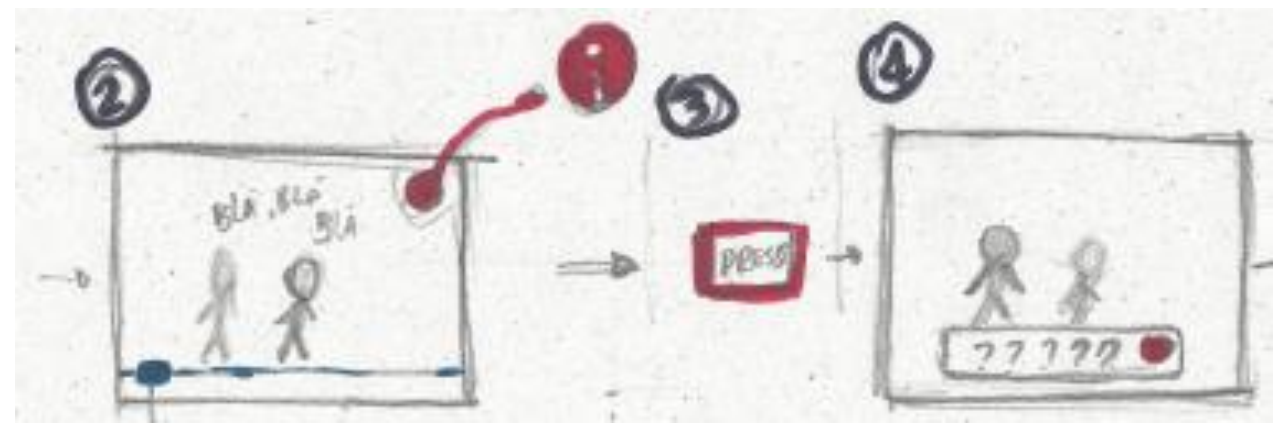

Figura 4. Recorte de linguagem pictórica

Logo abaixo do modelo mental da Figura 4, há uma descrição textual do comportamento do aplicativo (omitida por limitações de espaço), informando a ordem de apresentação das cenas (sequência de passos) e algumas propriedades relacionadas ao tempo (refletindo o conceito de âncoras da linguagem NCL), conforme resume-se na transcrição a seguir: "1. Iniciar o vídeo; 2 . Após 5 segundos, surge a mídia interativa; 3. Pressionar botão (vermelho) para interagir".

A combinação da linguagem pictórica com a linguagem verbal está presente em quase todos os casos analisados, contudo 03 participantes não utilizaram palavras para enriquecer seus modelos e apenas 01 as utilizou somente para reforçar a funcionalidade de algumas figuras.

Há um predomínio de estruturas similares em diversos modelos. Alguns exemplos são as "cenas interativas" (frame específico de um vídeo no qual a possibilidade de interação é visualmente explicitada) como, por exemplo, a representação de um botão vermelho na tela (Figura 4).

A Figura 4 mostra também uma distribuição espacial das mídias na tela, cujo conceito remete a utilização da visão de leiaute que algumas ferramentas de autoria possuem. No entanto, percebe-se que, nos modelos mentais analisados, há uma relação de navegação entre as diferentes visões (Figura 5), denotando a existência de relacionamentos causais (condição e ação) entre uma visão de leiaute e outra. 


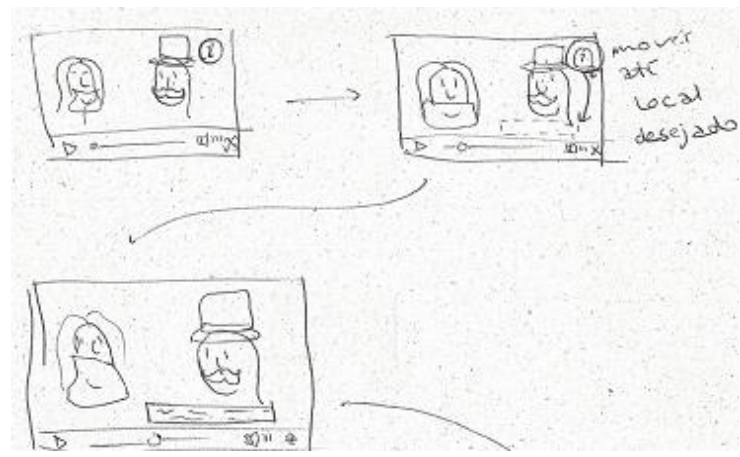

Figura 5. Relacionamentos entre cenas interativas

Alguns participantes fizeram uso do seu modelo mental para aprimorar o sistema empregando funcionalidades que não estavam no programa TVDi apresentado. A Figura 6 revela um exemplo de caso em que a simulação da nova funcionalidade foi verificada também nos participantes P1, P3, P4 e P9.

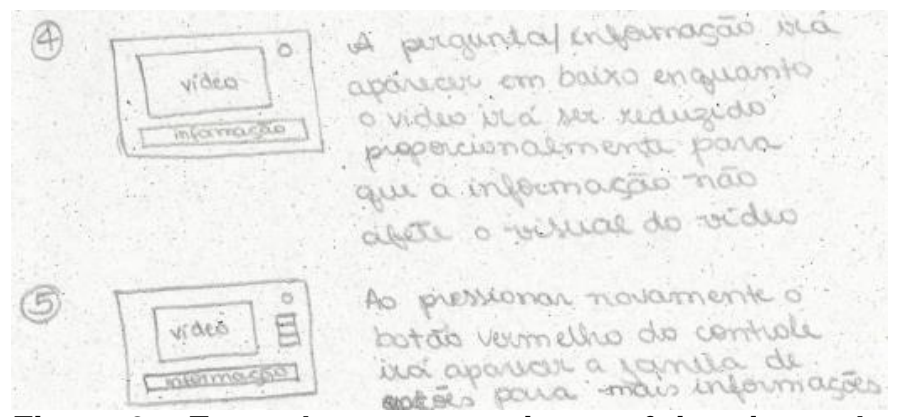

Figura 6. - Exemplo em que o sistema foi aprimorado

Conforme observa-se no fragmento do modelo mental da Figura 6, após alguns passos iniciais, o participante adicionou ao seu modelo a possibilidade de alterar as propriedades do vídeo principal, promovendo o seu redimensionamento. Isso sugere que o participante dominou seu modelo e simulou novas ações com base nele.

\section{Considerações Finais}

Os modelos mentais são naturalmente gerados para representar sistemas do mundo real. São naturalmente úteis para instanciarem execuções mentais tantas quantas se fizerem necessárias para que ocorra a compreensão de um dado sistema utilizado. Neste trabalho a validação do modelo mental ocorreu mediante as explanações dos participantes sobre os elementos estruturais, comportamentais e especiais de um OA.

Com este trabalho foi realizada uma verificação da estrutura cognitiva dos participantes (discentes de Design), cujos modelos mentais sugerem uma adoção coletiva de storyboards interativos como metáfora de representação devido a sua presença em diversos modelos apresentados pelos participantes. Notou-se também o emprego da linguagem natural (descrição textual dos elementos) como um possível modo de representação complementar para prover interação com a interface do OA.

A média geral dos modelos mentais foi de 63,77, sendo que apenas 06 (seis), dos 13 participantes válidos, superaram essa média. Isto é, esse grupo conseguiu compreender e aplicar adequadamente os conceitos intrínsecos aos elementos do aplicativo (comportamentais, estruturais e espaciais). Contudo, por se tratar de uma minoria, conclui-se que a estrutura cognitiva presente no aplicativo TVD $i$ desenvolvido, mesmo 
V Congresso Brasileiro de Informática na Educação (CBIE 2016)

Anais do XXVII Simpósio Brasileiro de Informática na Educação (SBIE 2016)

sendo de baixa complexidade em relação às poucas funcionalidades implementadas, não apresenta um nível trivial de compreensão pelos participantes desse teste. Enfatiza-se que os resultados são aplicáveis ao protótipo apresentado, todavia o processo de mensuração do modelo mental proposto pode ser estendido a outras aplicações, podendo apresentar um resultado distinto do obtido com este protótipo.

Em um nível mais detalhado os resultados informam que os participantes tiveram mais dificuldades para utilização dos conceitos básicos (média $=53,8$ ) do que para descreve-los (média $=73,5$ ) no aplicativo. Com base nesse resultado, reforça-se que a utilização de elementos presentes no aplicativo TVD $i$ desenvolvido é mais complexa do que a sua compreensão teórica - o que talvez seja resolvido com a realização de mais aulas sobre aplicativos dessa natureza.

Por fim, como trabalho futuro, pretende-se verificar a produção de novos modelos mentais após a refatoração do aplicativo aumentando-lhe a complexidade de relacionamentos e funcionalidades e implementar um novo OA para TVDi a partir de uma estrutura cognitiva previamente verificada.

\section{Referências}

Barbosa, S. D. J.; e Da Silva, B. S (2010), Interação Humano-Computador. Elsevier. Santa Rosa, J. G. e Moraes, A (2012). Avaliação e projeto no design de interfaces. Teresópolis, RJ: 2AB.

Borges, A. T.(2008), Como evoluem os modelos mentais. Ensaio Pesquisa em Educação em Ciências. Chaves, R. el. al. (2011),

DESIGMPS: Um Jogo de Apoio ao Ensino de Modelos de Qualidade de Processos de Software, baseado em Mapas Conceituais. In: XXXIX Congresso Brasileiro de Educação em Engenharia, 2011, Blumenau.

COBENGE. Damasceno, A. L. D. B. et. al. (2014). Cacuriá: Uma Ferramenta de Autoria Multimídia para Objetos de Aprendizagem. In Anais dos Workshops do Congresso Brasileiro de Informática na Educação.

Edwin, M. et. al. (2012). Multiplatform learning system based on interactive digital television technologies, IDTV. In Informatica (CLEI), 2012 XXXVIII Conferencia Latinoamericana. IEEE.

Miranda, S. et. al. (2015), Experimento de uma ferramenta de fórum baseado em mapas conceituais: uma nova forma de interação. In Anais do Simpósio Brasileiro de Informática na Educação.

Moraes, A. de; Santa Rosa, J. G. Avaliação e projeto no design de interfaces. Rio de Janeiro: 2AB-Série Oficina, 2012.

Moreira, M. A. (2000), Aprendizagem Significativa Crítica (Critical Meaningful Learning). Teoria da Aprendizagem Significativa.

Soares, L. F. S. e Barbosa, S. D. J. (2012), Programando em NCL 3.0 - Desenvolvimento de Aplicações para o Middleware Ginga - TV Digital e Web. 2nd. Ed. Rio de Janeiro - RJ: Editora Campus.

Tavares, R. (2006), Aprendizagem significativa em um ambiente multimídia.V Encuentro Internacional sobre Aprendizaje Significativo. 